\title{
Research on the Construction of Humanistic Environment of Library
}

\author{
Yingpei Wang Wa $^{1, \text { a }}$ \\ ${ }^{1}$ Library of Jilin Agricultural University, Changchun, Jilin, China, 130000 \\ ${ }^{a}$ email
}

Keywords: Library, Humanistic Environment, Construction Thoughts

\begin{abstract}
Good humanistic environment of a library can stimulate the readers' reading desire and narrow the distance between readers and the library. This paper firstly summarizes the connotation of the humanistic environment of the library, and then discusses the necessity of humanistic environment construction of the library, finally explores the construction thoughts of humanistic environment of the library to provide some references for the related researchers.
\end{abstract}

\section{Concept of Humanistic Environment of Library}

The humanities environment usually refers to various cultural phenomena of human society, is the sum of all material and spiritual achievements created by mankind in the process of reforming nature and society; special cultural environment, the formation refers to the human language, culture and ideology of various activities in the field of material and spiritual environment and atmosphere. In terms of the humanistic environment of the library, it is the invisible external environment, which is the direct or indirect effect and influence on the library, such as the quality of people, the interpersonal relationship, and the standard of the system, the internal and external environment. In advocating people-oriented service concept of the library today, the hardware condition of surface is only the humane environment, the connotation of Library's humanistic environment is reflected in the harmonious interpersonal relationship, learning atmosphere and warm to the reader itself from the heart care, respect, love, trust and so on. The service and management of library should be focused on the needs of readers, from the building to the layout, from the equipment to the opening time, from the service attitude to the service content, from the planning process and service setting, from management to the Department, to seek the maximum satisfaction of the reader, the reader needs to be fully respected. And meet. Library is the holy land of knowledge gathering, and it is an important place for the inheritance of civilization. The connotation of Library's humanistic environment refers to the library based on common occupation morality and value pursuit of continuous transformation which has characteristics of the humanistic spirit and culture of library can meet the internal and external environment, librarians and readers of material and spiritual needs of the environment.

\section{Necessity of the Construction of Humanistic Environment of Library}

The library should be based on the purpose of serving the readers, understanding the readers interested readers love readers, improving the humanistic environment can not only enhance the cultural quality of the whole library and school, more important is the virtuous cycle can form campus humanities environment construction. The library of every member in the environment are exerting influence, at the same time, each one was on the campus of the overall environment influence such as the good learning environment in the library is spacious and bright clean and orderly natural can attract more readers into the library reading and learning; at the same time, 
everyone can continue to forge ahead in learning atmosphere influence of learners in the library; after learning to develop good study habits, and can also affect other occasions learners, so in the environment can form a positive good style of study. In the face of the good environment of the library, the reader will not be wantonly destroyed, greatly reducing the occurrence of uncivilized behavior, but also can subtly reduce the campus of other places uncivilized behavior. Also, the library rules and regulations reasonably clear, warm service, a variety of Librarians' harmonious interpersonal relations, have played a role in moral education and behavior education make the reader a subtle normative behavior imperceptibly, enhance the realm of life and improve the quality of readers, and is convenient for the library staff management and communication, can eventually be better for reader service. This library gives full play to its own advantages in the human environment, in the leadership, librarians. Readers set up mutual understanding, mutual respect, and mutual trust of the bridge. It not only makes the humanistic environment of university library to enter a virtuous circle track, but also becomes an important force in the construction of the campus cultural environment.

Learning is the most important condition of modern people's survival and development, for college students, listening and reading is learning the most basic way: read high quality books, can obtain more accurate knowledge, solid, is more suitable for different cognitive style individuals. Reading must not be satisfied with general browsing, and must be a kind of study, develop good reading habits, make readers more carefully to the quality of life, the hole, to capture the benefits of knowledge and information in mind, nourish life from the wider world of life. Most of the students are willing to study in a quiet and harmonious environment in the library. First of all, the library with its unique quiet, quiet, sacred, and affinity of the human environment for college students to create a good reading atmosphere, so that it has a strong desire to read. Secondly, through the library recommend books, reading report and other activities, to guide students to establish a correct reading purpose and standard, and the hot spots around the various forms of reading instruction, make the students learn how to efficiently select useful books and materials. Finally, with the progress of science and technology, computer and multimedia technology is applied more and more widely in the library of university library by providing a user interface, extensive good retrieval function and intuitive man-machine interface, readers can query documents in the library by using computer network.

\section{Construction Thoughts of Humanistic Environment of Library}

Harmonious Humanistic Environment. The humanities environment construction of the library should pursue harmony, mainly displays in the harmony between the library and the natural environment and the two forms of the harmonious interpersonal relationship in the library. Generally speaking, the construction should be the first choice of beautiful landscape places of public library, the library of the strong elegant atmosphere of the school into the green landscape, fresh and elegant. Library not only harmonious integration into the surrounding environment, but also add to the green ecological environment of human color. The National Library uses the bamboo pole million is a natural model library and have all fallen into the trap, harmonious environment. University library not only to select the natural landscape of the beautiful, but also should be located in the campus center, to facilitate access to teachers and students. Library should not be built in the vicinity of the road or near the playground, should not be built in the vicinity of the school of music, fine arts, making it difficult to integrate into the surrounding environment of the surrounding environment. The location of the library embodies the pursuit of the harmonious humanistic environment of the library. The interpersonal relationship in the library includes the 
relationship between the manager and the librarian, the relationship between the librarian and the librarian, and the relationship between the librarians and the readers. The harmony between the librarians and the readers is the most important. Because of the work of the librarians are repetitive monotonous work, no innovation and challenge, the librarians work more than the production of burnout, the attitude of indifference to readers. Tea can help librarians to eliminate depression, open minded, willing to selfless dedication to the readers, close to readers with sincerity, create a harmonious atmosphere for the readers to read. In daily work, librarians should show professional dedication, pay attention to the use of the term civilization, can coordinate the relationship between librarians and readers. Librarian good attitude, good skills, friendly conversation, positive work attitude can create a harmonious humanistic environment.

Neat Humanistic Environment. We should keep the floor, reading tables and shelves clean and tidy, the appropriate window, keep indoor air flow, but also is to keep fresh, bright light and dark or when the weather is close to the evening lights should complement the light. This one week should at least make a sweep and a small sweep. Then in the neat shelf our books to do finishing work, processed in the book shelves before, should first check the category of books, classification number, and then according to the classification of books, the book neatly placed into the corresponding shelves. When usually free librarian should also regularly check the books on the shelf there is no wrong frame, disorder phenomenon, and timely to put the wrong books rearranged correctly, but also remember to often guide the reader to the correct use of indexes, to reduce the reader misplaced, scrambling of the books. The layout should follow the clean, efficient, safe, easy to operate and convenient readers' principle, such as the computer retrieval hall should be located in the library Front Gate hall or side hall is. In the hall should be set up publicity column, the introduction of the library rules and regulations, post library and readers of the circular and related publicity materials, to play a good guide role. Set up signs, signs, or paintings on the wall of a static library culture form. Interior decoration should be concise, highlight the theme of learning and knowledge, you can put some flowers and potted plants in order to enhance the sense of beauty and intimacy of the indoor environment, to eliminate the fatigue of the reader for a long time to learn.

Quiet Humanistic Environment. Quietness refers to the quiet environment, but also refers to the peace of mind. Library's humanistic environment construction should also pay attention to these two points, in the pursuit of quiet environment, but also the pursuit of peace of mind. The site selection should be avoided in the busy street, beside the noisy environment makes readers not meditation inside the library, the library knowledge dissemination function is difficult to play. The library can through a series of physical means, such as the sound insulation windows and sound-absorbing material in polymer, eliminate the external sound interference to beautify the environment. The sounds in the library can also affect other people's learning and reading. In the library should be labeled "turn your mobile phone to vibration or mute" and other words, to remind the reader to keep quiet environment. Peace of mind is the guarantee of the effect of learning and reading, the library has the obligation to help readers to build a quiet mind from the environment. The library should be on both sides of the road with a tree with luxuriant foliage, making the readers enjoy entering a cool, quiet attitude helps readers. Library to maintain appropriate temperature and humidity can make readers in the cold or hot weather quickly calm down to study and learn. In addition, the library placed green plants to ease the visual fatigue of readers. On the other hand, the ecological environment of green can make readers calm and improve the efficiency of reading.

Comfortable Humanistic Environment. As an important place for learning, the library should create a pleasant and comfortable humanistic environment for the readers. The humanistic 
environment, which is pleasant and comfortable, is first reflected in the quiet and elegant environment of the library. The conditional library is located in between the blue sky, a landscape of lakes and mountains besides studying can enjoy the surrounding scenery, some can be refreshing. The open reading methods, make readers swim in the ocean of knowledge, the fastest and most convenient access to the books is another meaning of the comfort of the humanistic environment of the library. Open access should embody the people-oriented spirit of service, the shelf distance should be moderate, so many people have no sense of the crowded shuttle in between the shelves of books; height should be moderate, easy access and access to the readers; librarian set as close as possible to the readers, between the closer the distance between the reader and the height of the counter at about $75 \mathrm{~cm}$, between the high prone to the reader's sense of alienation, is not conducive to the construction of a comfortable environment. Open type of lending allows readers to access a large number of books, but may be difficult to find a book to find. The library should set up the book location inquiry area, through the inquiry of the computer, it can determine the exact position of the book, reduce the search time of the reader, and enhance the reader's reading pleasure. Open type of lending should be at the same time with the "wide into the strict out" in the way of access. Library allows readers to carry bags into the museum, to facilitate the reader to learn. For readers to leave the library, through electronic equipment strictly investigate and deal with books without lending out of the library. Librarians in dealing with the problem should be a kind attitude, full respect for the reader. The library should install the barrier free design, open wheelchair access, and the distance between the library shelves should meet the requirements to provide special wheelchairs, blind books to vulnerable groups of vulnerable groups to enhance reading right, reading pleasure. In addition, providing a wireless network and the necessary public computer for readers to access information also helps to create a pleasant and comfortable human environment.

\section{Conclusion}

Since the twenty-first century, the library cause in our country has also presented a vigorous development trend. The humanistic spirit is the eternal pursuit of the library. The construction of the humanistic environment of the library is an important way to improve the level of scientific management and service. The library should make great efforts to create a harmonious, neat, quiet and comfortable humanistic environment to serve the readers better.

\section{References}

[1] Liu Shiping, Chen Yan, Journal of Hunan University of Science and Engineering, Vol. 31 (2010) No 10, p.205-207

[2] Long Yanchun, Chen Haikun, Journal of Yunnan Finance and Trade Institute, Vol. 17 (2011) No 1, p.210-211

[3] Liu Ying, Journal of Tianjin Academy of Educational Science, Vol. 25 (2013) No 3, p.28-30

[4] Long Qu, Sichuan University of Arts and Science Journal, Vol. 24 (2014) No 5, p.158-160 